\title{
Imaging features of mycotic aortic aneurysms
}

\author{
Nan Zhang" ${ }^{1 \#}$, Wei Xiong ${ }^{2 \#}$, Yu Li ${ }^{1}$, Qinxiang Mao ${ }^{3}$, Shangdong $\mathrm{Xu}^{4}$, Junming Zhu ${ }^{4}$, Zhonghua Sun ${ }^{5}$, \\ Lizhong Sun ${ }^{4}$
}

${ }^{1}$ Department of Radiology, Beijing Anzhen Hospital, Capital Medical University, Beijing, China; ${ }^{2}$ Department of Respiration, First Teaching Hospital of Tianjin University of Traditional Chinese Medicine, Tianjin, China; ${ }^{3}$ Department of Radiology, Longtan Hospital of Guangxi Zhuang Autonomous Region, Liuzhou, China; ${ }^{4}$ Department of Cardiovascular Surgery, Beijing Aortic Disease Centre, Beijing Anzhen Hospital, Capital Medical University, Beijing Institute of Heart Lung and Blood Vessel Diseases, Beijing Engineering Research Centre for Vascular Prostheses, Beijing, China; ${ }^{5}$ Discipline of Medical Radiation Science, Curtin Medical School, Curtin University, Perth, Australia

\#These authors contributed equally to this work as co-first author.

Correspondence to: Professor Yu Li. Department of Radiology, Beijing Anzhen Hospital, Capital Medical University, Chaoyang District, Anzhen Road 2nd, Beijing 100029, China. Email: 1523115105@qq.com; Prof Zhonghua Sun, Discipline of Medical Radiation Science, Curtin Medical School, Curtin University, Perth, 6102, Australia. Email: z.sun@curtin.edu.au.

\begin{abstract}
Infectious aortitis (IA) is a rare and life-threatening cardiovascular disease. Early diagnosis and timely intervention are crucial for reducing mortality associated with mycotic aortic aneurysms (MAAs); however, early diagnosis is challenging due to the nonspecific symptoms. Some cases are diagnosed at an advanced stage or after developing complications, such as rupture or aortic fistula. Current state-of-theart imaging modalities_-including computed tomography (CT), magnetic resonance imaging (MRI), and 18F-fluorodeoxyglucose (FDG) positron emission tomography (PET)/CT—can detect infected aneurysms in clinically suspicious cases. MAA features on imaging include lobulated pseudoaneurysm, indistinct irregular arterial wall, perianeurysmal gas, perianeurysmal edema, perianeurysmal soft tissue mass, aneurysmal thrombosis, and high metabolic activity with increased uptake of FDG. Enlarged lymph nodes are often found adjacent to the aneurysm, while iliopsoas abscess (IPA), spondylitis, and aortic fistulas are commonly associated complications. After surgery or endovascular repair, radiological features-including ectopic gas, peri-graft fluid, thickening of adjacent bowel, pseudoaneurysm formed at the graft anastomosis, and increased uptake of FDG-may indicate an infection of aortic graft. This article provides an overview of the clinical and imaging features of MAAs. Thus, familiarity with the imaging appearances of MAAs may assist radiologists in the diagnosis and facilitation of timely treatment.
\end{abstract}

Keywords: Infection; aorta; mycotic aneurysm; computed tomography; imaging

Submitted Aug 03, 2020. Accepted for publication Dec 23, 2020.

doi: 10.21037/qims-20-941

View this article at: http://dx.doi.org/10.21037/qims-20-941

\section{Introduction}

Infectious aortitis (IA) is a rare, life-threatening cardiovascular disease for which early diagnosis can be missed due to a lack of specific clinical, radiological, and laboratory features. IA's common sign is a mycotic aortic aneurysm (MAA), which manifests as a mushroom-shaped structure on a blood vessel. This manifestation does not refer to a specific pathogenic cause, such as a fungal infection (1); rather, MAAs is an acute inflammatory response to pathogenic infection, which induces neutrophilic infiltration at the arterial wall. During this process, the collagenolytic and elastolytic enzymes are activated, which is concomitant with saccular lumen dilation and rupture (2-4).

MAAs are associated with high mortality due to their increased risk of rupture, which is especially common in the 
abdominal aorta compared with peripheral arteries (5). Early diagnosis and timely intervention are critical in reducing the mortality of MAAs; however, early diagnosis is challenging due to nonspecific symptoms and low sensitivity of blood cultures (6-9). Furthermore, the incidence of adverse events in patients with MAAs after invasive treatment is higher than those with pseudoaneurysms associated with other causes, such as trauma and atherosclerosis. The endovascular stenting of MAAs may be associated with a high risk of stent infection, endoleak, reinfection, and potential rupture $(10,11)$.

The diagnosis of MAAs requires awareness of the spectra of computed tomography (CT), magnetic resonance imaging (MRI), and ${ }^{18} \mathrm{~F}$-fluorodeoxyglucose (FDG) positron emission tomography (PET)/CT. The use of CT angiography (CTA) in the assessment of aortic diseaseincluding MAAs-is increasing, owing to its non-invasive, efficient, broad coverage and its isotropic voxel capabilities. With CT technology development and advanced dose reduction techniques, CTA is a fast and high-quality method with minimal contrast medium and radiation dose. The high tissue resolution of MRI can provide valuable anatomical and physiological information, especially in assessing abscess and tissue edema. In the latest studies, ${ }^{18}$ F-FDG-PET/CT has shown higher sensitivity and diagnostic accuracy in infected aortic aneurysms and aortic prosthetic graft infection compared to CTA $(12,13)$.

This review provides an overview of the clinical, pathological, and radiological presentations of MAAs. The imaging findings during the period following medication, interventional, or surgical management are also described. The imaging characteristics in other infectious pathogens are highlighted for differential diagnosis, especially in patients with negative blood culture results.

\section{Pathogenesis}

MAAs usually occur in the elderly, predominantly affecting immunocompromised patients, such as diabetes mellitus, liver cirrhosis, end-stage renal disease, alcoholism, chronic glucocorticoid therapy, post-transplantation immunosuppression, human immunodeficiency virus infection, drug abuse, and malignancy (14-17). The known causative organisms of MAAs are Salmonella, Staphylococcus aureus, Klebsiella pneumoniae (KP), Escherichia coli, Mycobacterium, and Brucella melitensis. Fungi, such as Candida albicans and Aspergillus, are also rare causes of infected aneurysms.

\section{Salmonella}

Non-typhoidal Salmonella has been reported as the most common organism in Eastern countries, especially in the atherosclerotic abdominal aorta $(8,16,18-20)$. Salmonella usually resides in the phagosomes of host macrophages and other antigen-presenting cells, such as dendritic cells, which participate in the formation of atherosclerotic plaques. The immunocompromised condition contributes to its reproduction and invasiveness from atherosclerotic plaques (21-23) (Figure 1).

\section{Klebsiella pneumoniae (KP)}

Klebsiella infection can occur in almost all organs but is most commonly observed in the liver and lungs. Approximately $60-93 \%$ of patients with KP have comorbid diabetes mellitus $(15,24,25)$. KP can invade the aortic wall and induce MAAs from the location of damaged vascular endothelial walls (16). In patients suffering from aortic pseudoaneurysm and pyogenic liver abscess and who have a history of diabetes mellitus, $\mathrm{KP}$ should be considered as the causative organism (Figure 2).

\section{Mycobacterium tuberculosis}

About $75 \%$ of MAAs caused by Mycobacterium tuberculosis present as a contiguous lesion on the surrounding tissue, such as lymph node enlargement or paraspinal abscess $(26,27)$. Constant surveillance imaging can indicate pathogenesis (Figure 3).

\section{Staphylococcus aureus}

Pseudoaneurysm caused by Staphylococcus aureus has been reported in intravenous drug users, as well as iatrogenic or traumatic arterial wall injury patients $(28,29)$ (Figure 4). In these patients, MAAs develop from direct infectious inoculation at the time of vascular trauma.

\section{Brucella melitensis}

Brucella melitensis is a zoonotic intracellular Gram-negative coccobacillus responsible for multisystem infections, including the aorta-especially in immunocompromised patients. The common transmission route is through direct contact with infected cattle via milk or other body fluids (30). 

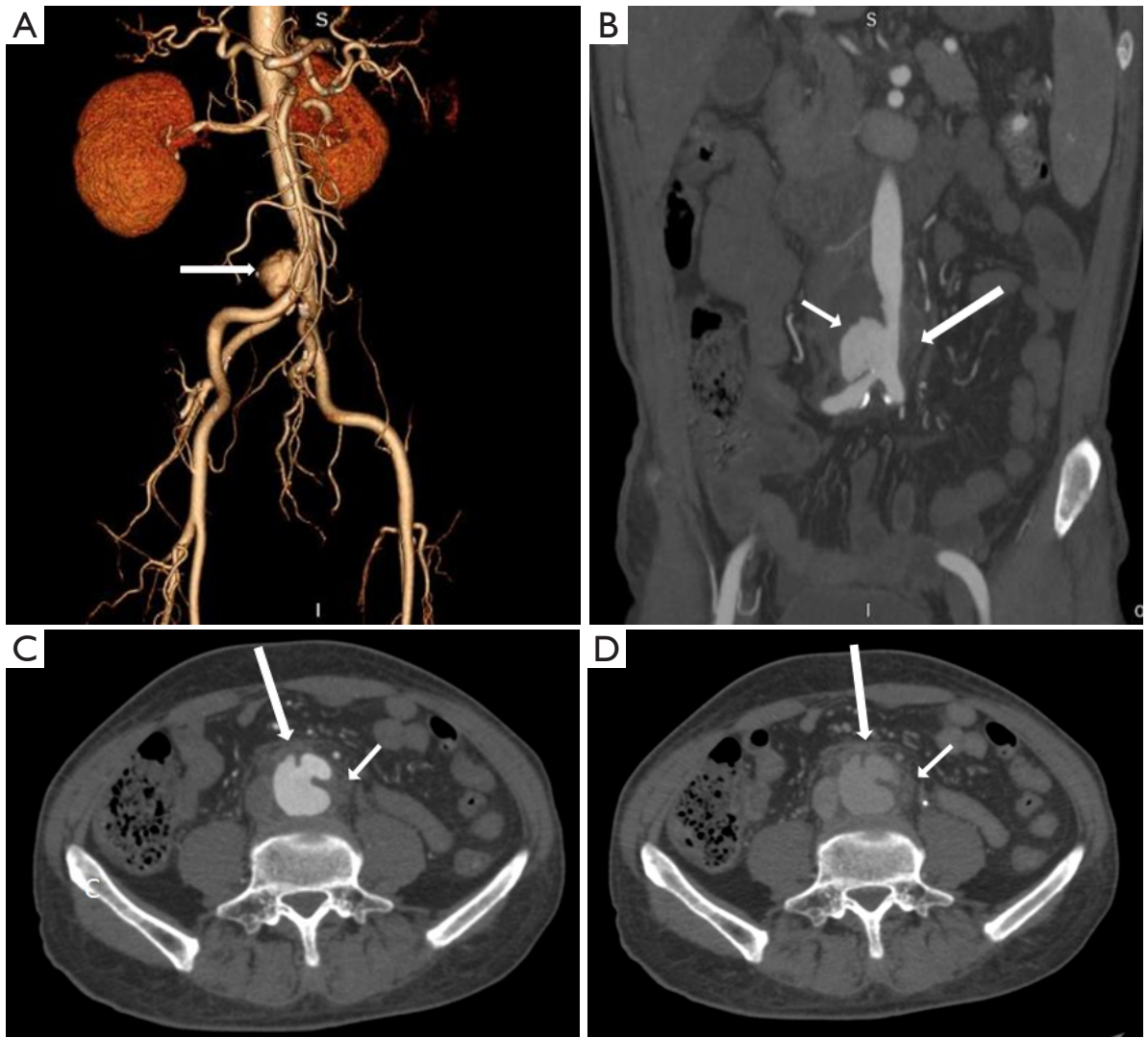

Figure 1 MAAs caused by Salmonella. A 58-year-old man with a history of the human immunodeficiency virus (HIV) and cirrhosis presented with fever and increasing abdominal pain. Salmonella was captured in hemocultures. (A) Three-dimensional (3D) CT angiography of abdominal aorta shows saccular pseudoaneurysm formation (arrow). (B) Coronal CT view shows mycotic aneurysm with periaortic soft tissue (long arrow) and mural thrombus (short arrow). (C,D) Transverse view (arterial and venous phases) shows disruption in the aortic wall. Periaortic soft tissue can be enhanced in the venous phase (long arrow). Lymph nodes are enlarged (short arrow). MAAs, mycotic aortic aneurysms.

\section{Clinical features}

Due to the risk of rapid expansion and consequent rupture, timely diagnosis and treatment of MAAs are paramount. Unfortunately, some patients could be clinically silent until an aneurysm rupture. The most frequent presenting symptoms of MAAs are fever and pain $(16,19)$. MAAs localized to the thoracic aorta usually manifest as chest pain, whereas infected abdominal aortic aneurysms usually manifest as abdominal pain with or without a pulsatile mass. The laboratory abnormalities are often nonspecific and may include elevated erythrocyte sedimentation rate (ECR), C-reactive protein (CRP), and leukocytosis. Blood cultures fail to detect bacteria in approximately $25 \%$ of cases, which might contribute to broad-spectrum antibiotic therapy administration.
Life-threatening hemorrhage-such as hemoptysis, gastrointestinal hemorrhage, and sequentially shock-could result from fistulae. The poor prognosis of these patients emphasizes the importance of early diagnosis. Aortoenteric fistula is caused by aneurysm infection spreading to the enteron, which usually begins at the duodenum, adjacent to the aorta (Figure 5). Thoracic MAAs with surrounding lung parenchyma infection or compaction cause aortobronchial fistula (Figure 6), a less recognized complication of abdominal MAAs. A dramatic increase in mortality occurs in patients without clear preoperative diagnosis compared to those with clear preoperative diagnosis $(100 \%$ in patients without a clear diagnosis $v s .15 \%$ in patients with clear diagnosis).

Iliopsoas abscess (IPA) is a common complication in abdominal MAAs with or without endograft infection, 

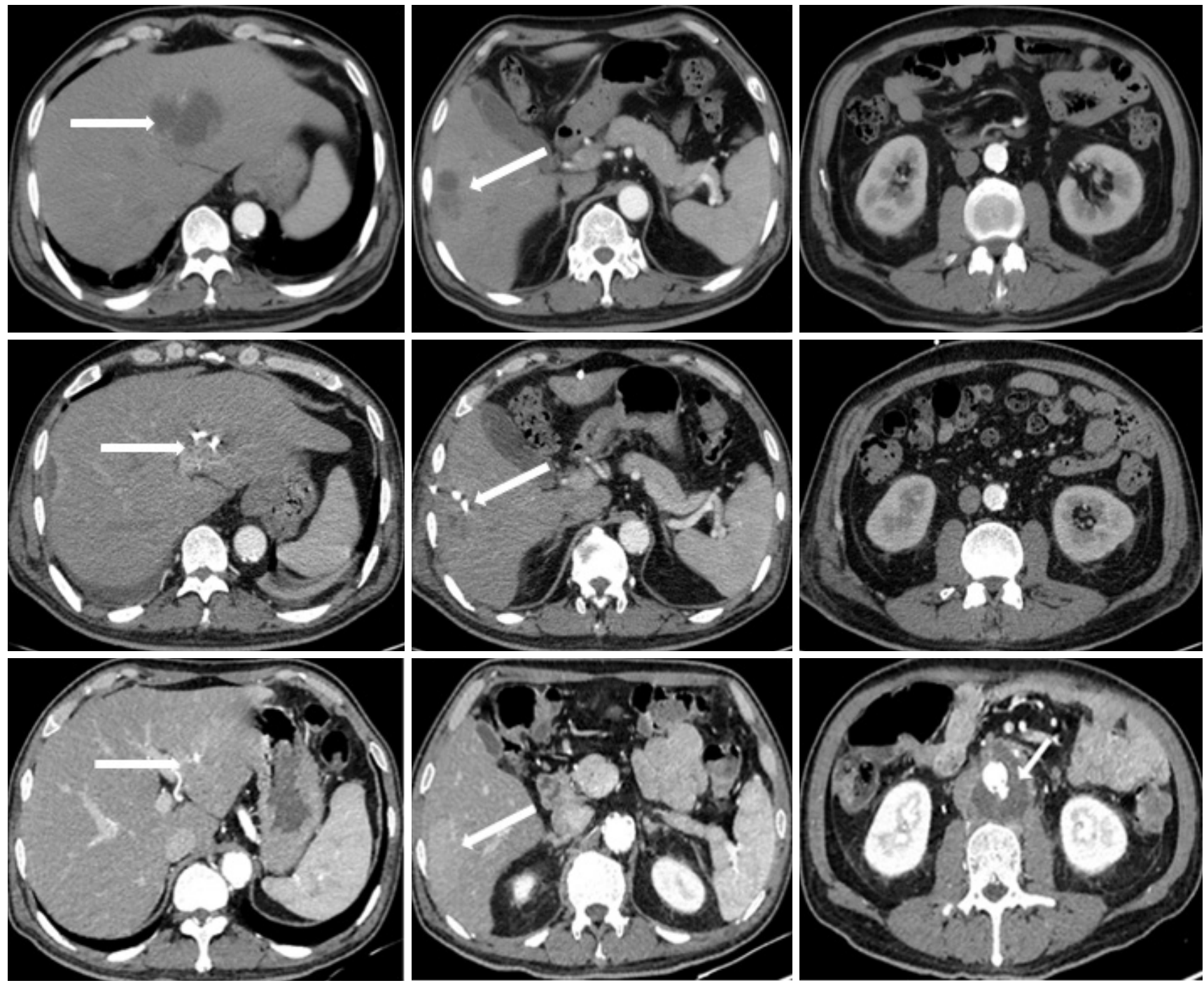

Figure 2 MAAs caused by Klebsiella pneumoniae. A 52-year-old male with a history of diabetes mellitus presented with fever $\left(39^{\circ} \mathrm{C}\right)$, chills, nausea, and abdominal pain. Blood culture of KP. Top row images: contrast-enhanced CT images obtained at 15 days after symptom onset. Multiple cystic lesions (in S4 and S6 of the liver; arrows) with contrast-enhanced septum and wall demonstrate liver abscess. No signs of aneurysm or inflammation around the abdominal aorta. Middle row images: contrast-enhanced CT images obtained 4 days after the drainage of liver abscess. The abscess became smaller after drainage and antibiotic treatment. Bottom row images: contrast-enhanced CT images obtained 35 days after the drainage of liver abscess (the patient relapsed 15 days after discharge). An aneurysm was found at the original abscess focus (long arrow in the middle image). The saccular MAA showed surrounding soft tissue (short arrow). MAA, mycotic aortic aneurysm.

presenting as a direct invasive infection with purulent materials occurring within the iliopsoas muscle. The causative organisms include Mycobacterium, Salmonella, KP, other Gram-negative bacilli, and mixed bacteria. The mortality rate is as high as $100 \%$ if IPA is left untreated (31-33). IPA is reported as a major risk factor for patients with MAAs; thus, clinicians should be cautious about this potential complication. In tuberculous spondylitis patients, MAAs can involve secondary spread from spine lesions (Figure 7). The rate of mortality is high among MAA patients with combined pyogenic spondylitis (34,35). 

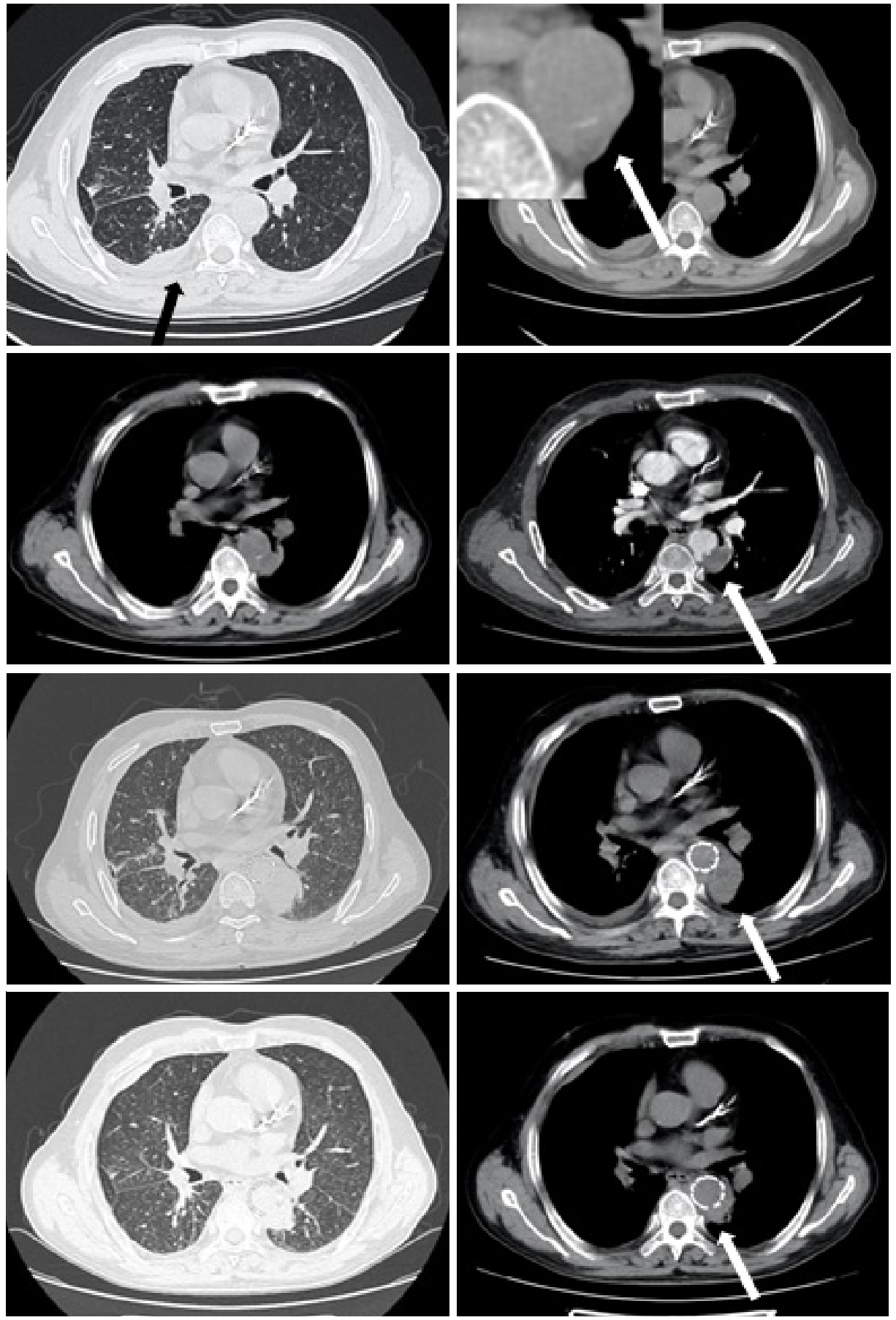

Figure 3 MAAs caused by Mycobacterium tuberculosis. A 72-year-old male repeatedly presented over 9 months with fevers. First-row images: CT images in January 2018 showed multiple miliary nodules and peripheral reticulation in bilateral lungs. Right pleural effusion and pleural thickness (black arrow), coronary artery calcification, and small lymph nodes adjacent to the posterior wall of the thoracic aorta are marked (white arrow). Second-arrow images: contrast-enhanced CT images in March 2018 demonstrated a small pseudoaneurysm adjacent to the enlarged lymph nodes (arrow). Third-row images: CT images in April 2018 showed stent graft and lymph nodes enlarged more than previously, with hypodense center indicating necrosis (arrow). Fourth-row images: CT images in October 2018 (standard antitubercular drug treatment for 6 months) showed partially absorbed lung nodules and pleural effusion. The size of lymph nodes decreased significantly (arrow). MAAs, mycotic aortic aneurysms. 

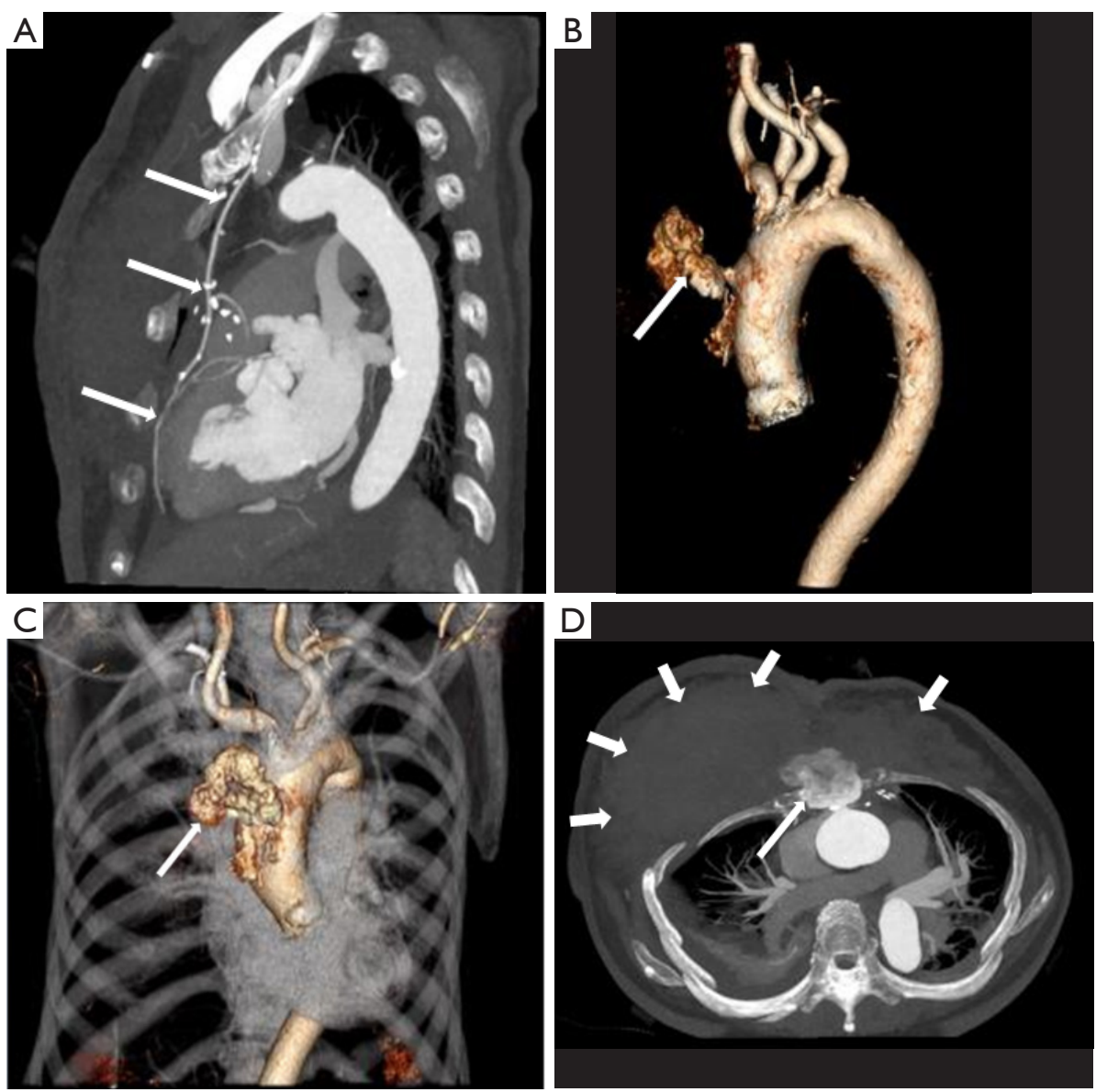

Figure 4 MAAs caused by Staphylococcus aureus. A 66-year-old woman presented with fever and increasing chest pain with a history of coronary artery bypass grafting performed before 6 months. Blood culture of Staphylococcus aureus. (A) CTA demonstrates the patency of the left descending artery bypass graft (arrow), (B,C) Irregular and lobular aneurysm arises from the ascending aorta (arrow). (D) The aneurysm ruptures into the anterior chest wall (arrow) with extensive soft tissue thickening (short arrows). CTA, computed tomography angiography; MAAs, mycotic aortic aneurysms.

\section{Clinical management}

Open surgical repair has been regarded as an effective treatment for MAA but is associated with a mortality rate of over $20 \%(17,29)$. The endovascular repair of MAAs-an expeditious temporization of MAA rupture in hemodynamic instability cases-is well-established. Kan et al. demonstrated that there was no significant difference in overall survival rate between open surgical repair and endovascular repair (20); however, the insertion of an endovascular graft in an infected field remains a major concern. Stent graft implantation is a significant independent predictor of persistent infection (20). Prolonged culture-specific antibiotic therapy, combined with open surgery or endovascular techniques, is a key component for successfully treating MAAs.

\section{Imaging features}

CT, MRI, and PET/CT are the most commonly used imaging modalities in the detection and assessment of clinically suspected infected aneurysms. Due to the higher quality spatial resolution of contrast-enhanced CT and MRI, these modalities can provide valuable information regarding the morphology of aortic aneurysm, aortic wall enhancement, and the relationship between the aneurysm and adjacent tissue; however, PET/CT is the most sensitive of these modalities in detecting infection, which is depicted by increased uptake of FDG. Like PET/CT, diffusion- 

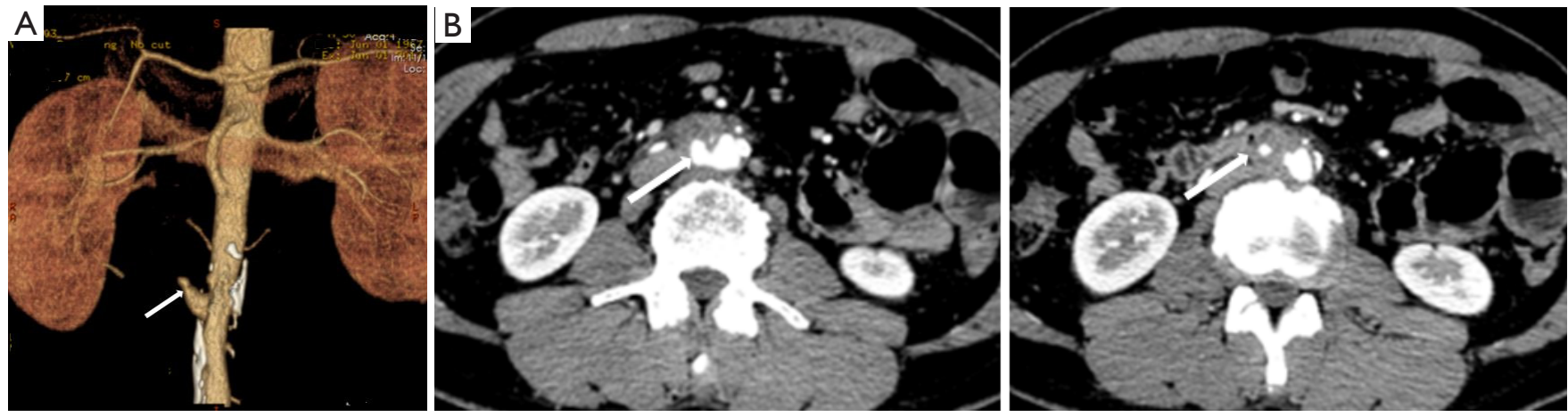

Figure 5 Aortoenteric fistula. A 50-year-old male with a history of diabetes mellitus presented with fever, abdominal pain, and melena. Blood culture showed the growth of Salmonella. (A) 3D CTA shows irregular MAA (arrow). (B) Transverse view demonstrates the interruption of the aortic wall and saccular pseudoaneurysm formed with communication between the aorta and the duodenum (arrow). MAA, mycotic aortic aneurysm.
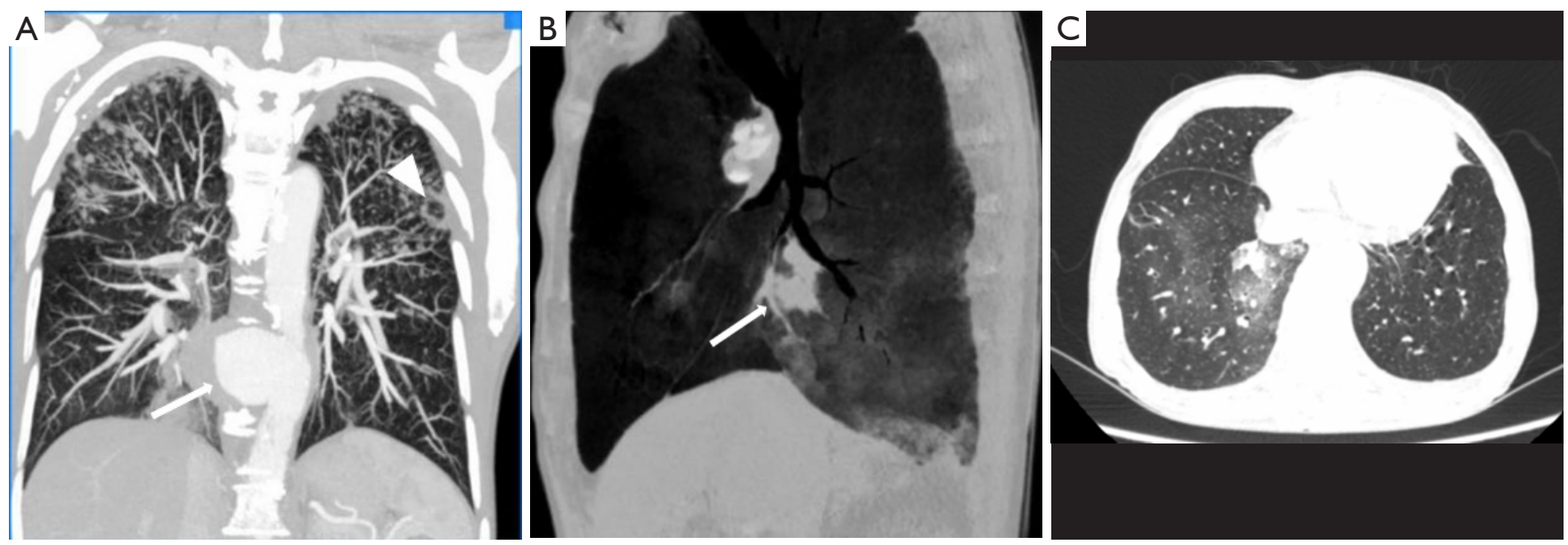

Figure 6 Aortobronchial fistula. An 84-year-old male with a history of diabetes mellitus and tuberculosis presented with fever, chest pain, and hemoptysis. (A) Maximum-intensity projection image shows multiple small nodules with sharp edges and upper lobe distribution, cavity (arrowhead), saccular pseudoaneurysm of the thoracic aorta with surrounding soft tissue (arrow), and patchy shadow in the right lower lobe. (B) Minimum-intensity projection image shows the lumen of right endobasal segmental bronchus (adjacent to the aneurysm wall) obstruction filled with high density material. (C) Patchy ground glass opacity and consolidation consistent with alveolar hemorrhage.

weighted imaging (DWI) MRI is also sensitive in detecting infection with restricted diffusion manifestation. DWI and T2-weighted MRI can assist in detecting soft tissue edema and adjacent organ involvement.

\section{Infected aortic aneurysm}

An infected aortic aneurysm appears on CT and MRI as a focal, contrast-enhancing, lobulated, saccular lumen, with an indistinct, irregular aortic wall (Figure 8). CT is the most sensitive imaging modality for detecting calcification and gas bubbles. The calcification interruption indicates the location of the disrupted aortic wall. Gas bubbles that appear in and around MAAs have high diagnostic reliability of etiology (Figure 9). Rapidly progressive growth of true or false aneurysms ( $>5 \mathrm{~mm}$ in 2 weeks) is also suggestive of an infectious etiology (Figure 10). The thickened MAA wall usually appears as a high signal intensity on T2-weighted MRI and DWI (Figure 11), with increased uptake of FDG on PET/CT. 

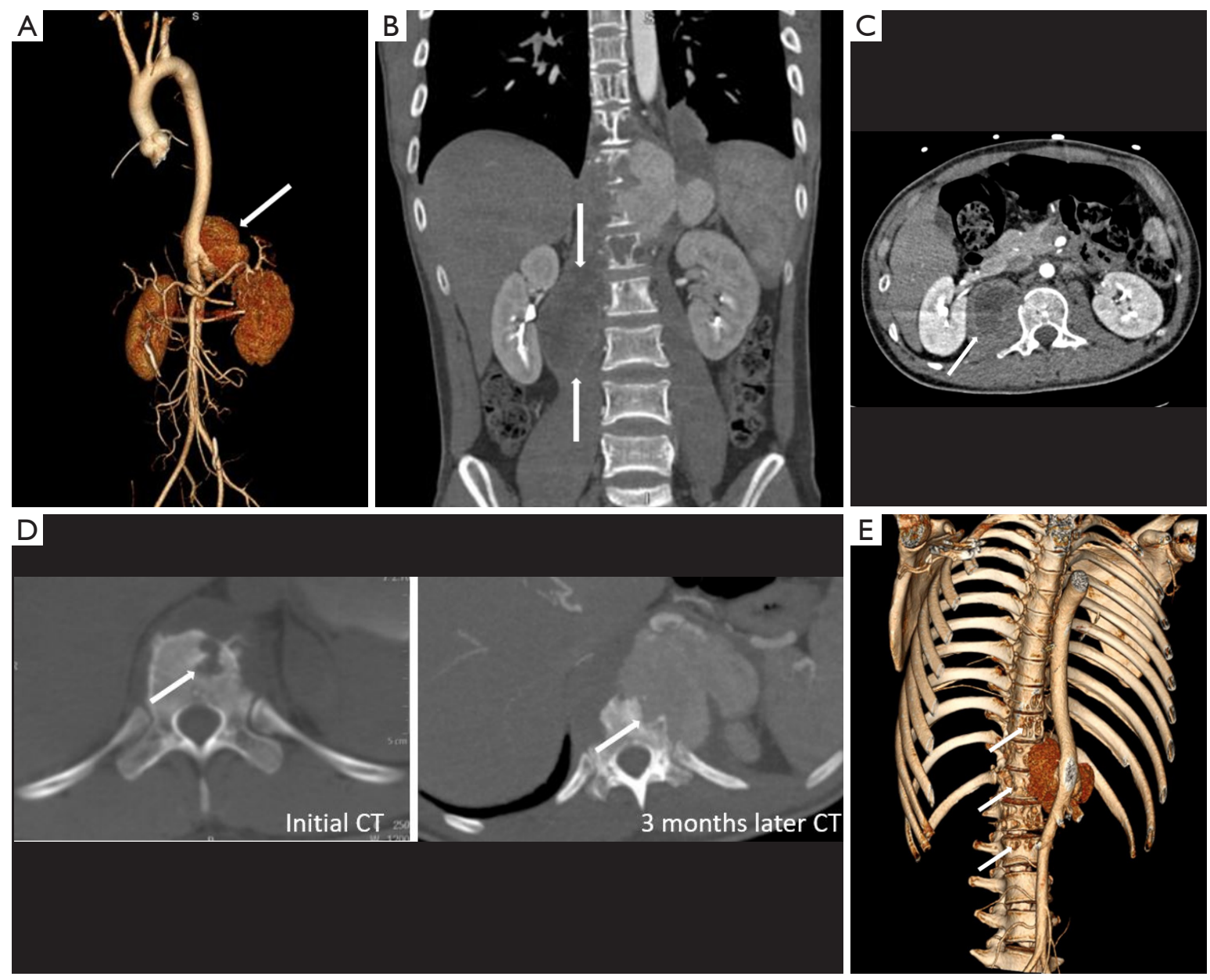

Figure 7 Tuberculous spondylitis involving the aorta, with IPA and MAA formation. A 20-year-old male with a three-month history of back pain, low fever $\left(37.5-37.9^{\circ} \mathrm{C}\right)$, and a further acute episode of back pain aggravated in recent days. (A) 3D CTA shows giant irregular MAA (arrow). (B,C) Right iliopsoas muscle is enlarged and swollen with a relatively low-density area and contrast-enhanced rim of the abscess wall (arrows). (D) Initial spinal CT imaging shows erosion of the vertebral body and endplate at T11 (arrow) with soft tissue swelling or abscess around the vertebral body. The outline of the aorta is distinct. CTA after 3 months shows the progression in the vertebral body's destructive changes (arrow), obliteration of fat planes between the vertebral body and aorta, and lobular pseudoaneurysm formed adjacent to the eroded vertebral body. (E) 3D CTA shows multiple lesions in the vertebral body at T9-L2 and pseudoaneurysm. CTA, computed tomography angiography; IPA, iliopsoas abscess; IPM, iliopsoas muscle; MAA, mycotic aortic aneurysm.

\section{Periaortic tissue}

Eccentric periaortic inflammatory soft tissue usually manifests as rim or septum enhancement following the administration of contrast material (venous phase) on contrast-enhanced CT and MRI (Figure 8). Periaortic edema appears as a distinctive fat stranding on CT; lymph nodes adjacent to MAAs might also appear swollen and enhanced (Figure 1). The edema of periaortic tissue and lymph nodes have the same MRI and PET/CT findings as the thickened MAAs wall described above (Figure 11).

\section{Adjacent organs}

CT provides a definitive diagnosis of IPA and other features that involve adjacent structures. IPA's typical features on CT include enlarged iliopsoas muscle, single or multiple relatively low-density abscess cavities with rim-like contrastenhanced walls (Figure 7), and in some cases, gas within lesions. Interestingly, MRI and PET/CT are sensitive modalities in detecting IPA with typical restricted-diffusion manifestation on MRI and increased uptake of FDG on PET/CT (Figure 11). 

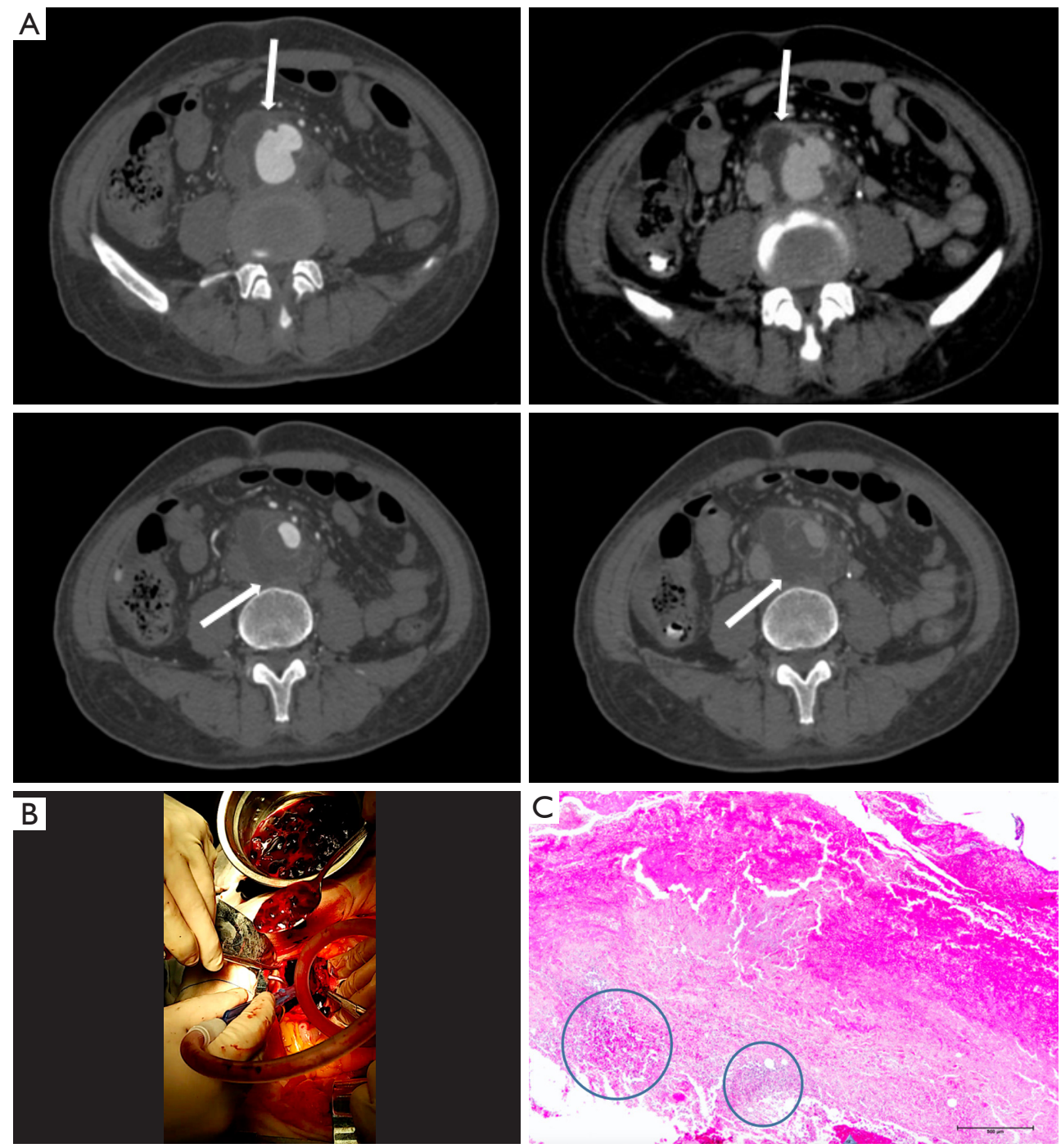

Figure 8 Morphology of MAAs. (A) Top row images: contrast-enhanced CT images show disruption to the aortic wall and saccular MAA with rim enhancement of periaortic thickened soft tissue (arrows). (A) Bottom row images: Low-density periaortic soft tissue indicates necrosis and abscess (arrows). The left posterior wall is intact, and a mural thrombus is observed in the lumen. (B) Intraoperative findings identified stagnant abscess around the infected aorta. (C) The hematoxylin-eosin (H\&E)-stained section of the infected aortic wall exhibits inflammatory infiltration with microabscess formation (circles) and loss of normal media architecture. MAAs, mycotic aortic aneurysms.

Primary and secondary pyogenic spondylitis manifests intervertebral disc and/or vertebral body destruction on CT and MRI (Figure 12). The diagnosis of pyogenic spondylitis can also be confirmed on PET/CT with increased metabolic manifestations. MRI and PET/CT enable spondylitis visualization without morphological changes in the early stage (Figure 11).

Contrast medium shunt from the aorta to the inferior vena cava, digestive tract, or bronchus, is a diagnostic sign for aortic fistula $(36,37)$. The shunt usually manifests as early enhancement of the inferior vena cava well before it appears in the renal and hepatic parenchyma, with a density of the adjacent digestive tract or bronchus similar to that of the adjacent aorta. When aortacaval fistula occurs, dilated and retrograded enhanced renal or iliac veins can be seen on enhanced CT and MRI, with direct communication between the aorta and inferior vena cava (Figure 13). Aortocutaneous fistulas are extremely rare and are usually seen in cases involving prior vascular prosthetic graft insertion (Figure 14) (38). Abnormal accumulation 

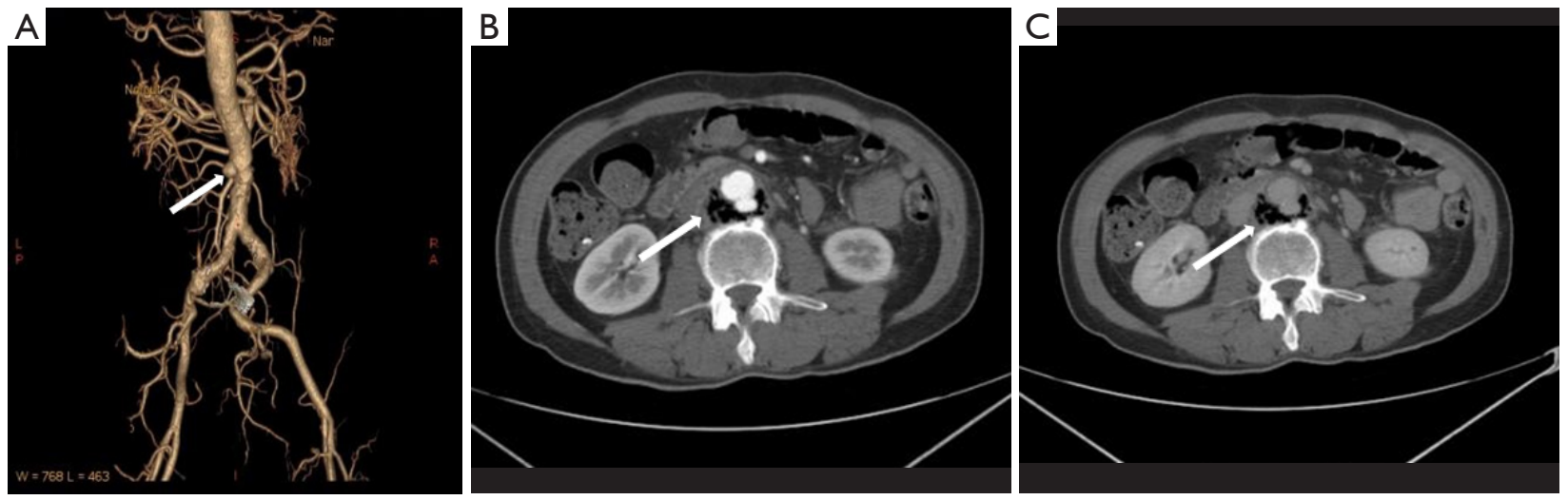

Figure 9 Air bubble sign. A 63-year-old male with a history of diabetes mellitus presented with fever and abdominal pain. Blood cultures consisted of Salmonella. (A) 3D CTA shows saccular MAA at the abdominal aorta (arrow). (B,C) Transverse contrast-enhanced CT images show an eccentric saccular MAA with posterior circumferential gas around the abdominal aorta. CTA, computed tomography angiography; MAA, mycotic aortic aneurysm.
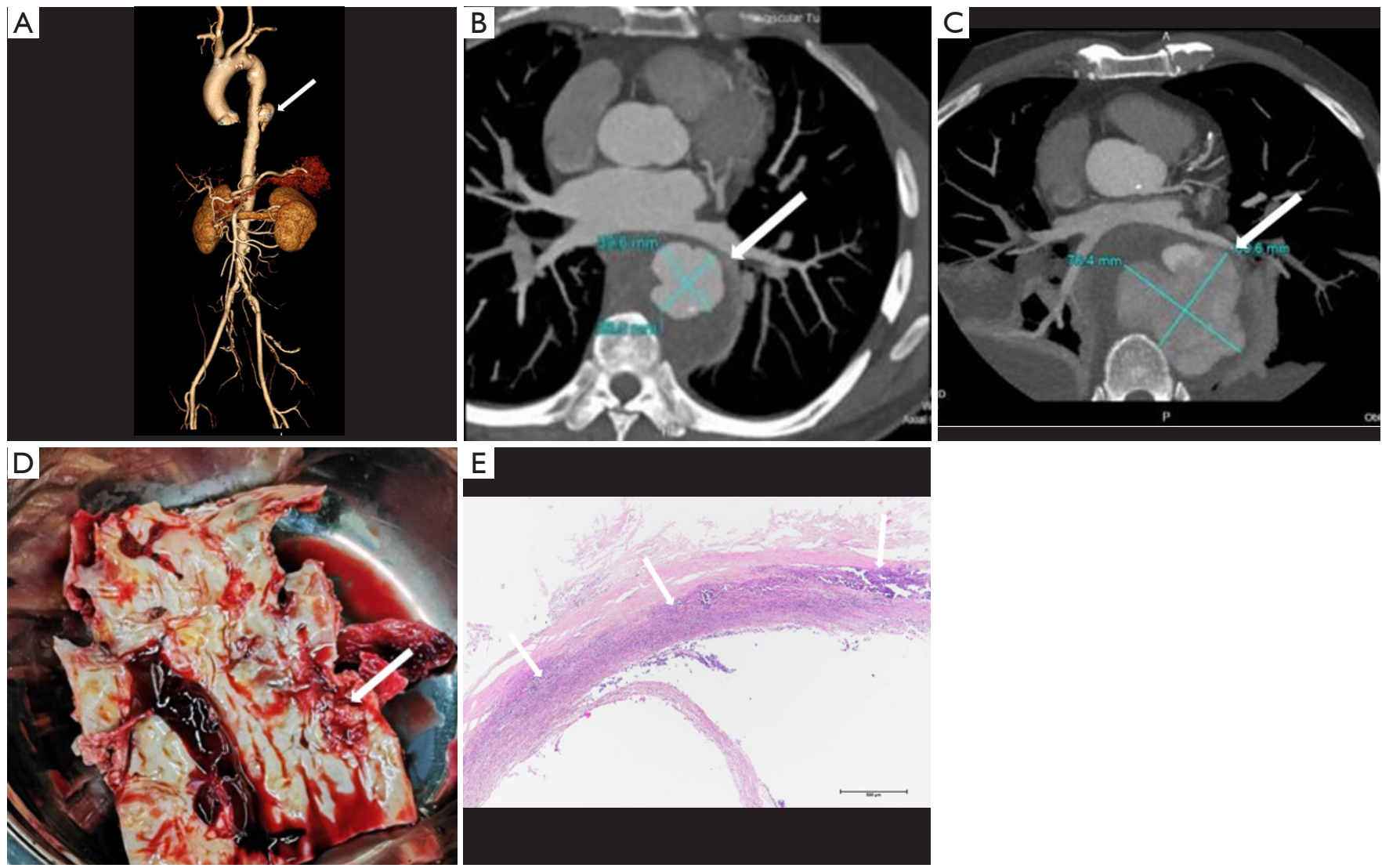

Figure 10 The rapid progress of MAAs. A 56-year-old male with a history of diabetes mellitus presented with fever and chest pain. Salmonella was detected in blood cultures. (A) 3D CTA of the aorta shows irregular MAA (arrow). (B) Transverse CTA image demonstrates the saccular aneurysm $\left(4.0 \times 3.9 \mathrm{~cm}^{2}\right)$. (C) The maximal caliber of the aneurysm increased to $7.6 \times 6.1 \mathrm{~cm}^{2}$ on the 15 -day follow-up. The left atrium is compressed (arrow). Bilateral pleural effusion indicates the fragility of the aneurysm wall. (D) The macroscopic finding of the MAAs shows severe atherosclerosis and mural thrombus in the lumen. The arrow shows the aortic destruction. (E) H\&E-stained samples show diffuse inflammatory infiltrate (arrows) and loss of normal media architecture. CTA, computed tomography angiography; MAAs, mycotic aortic aneurysms. 

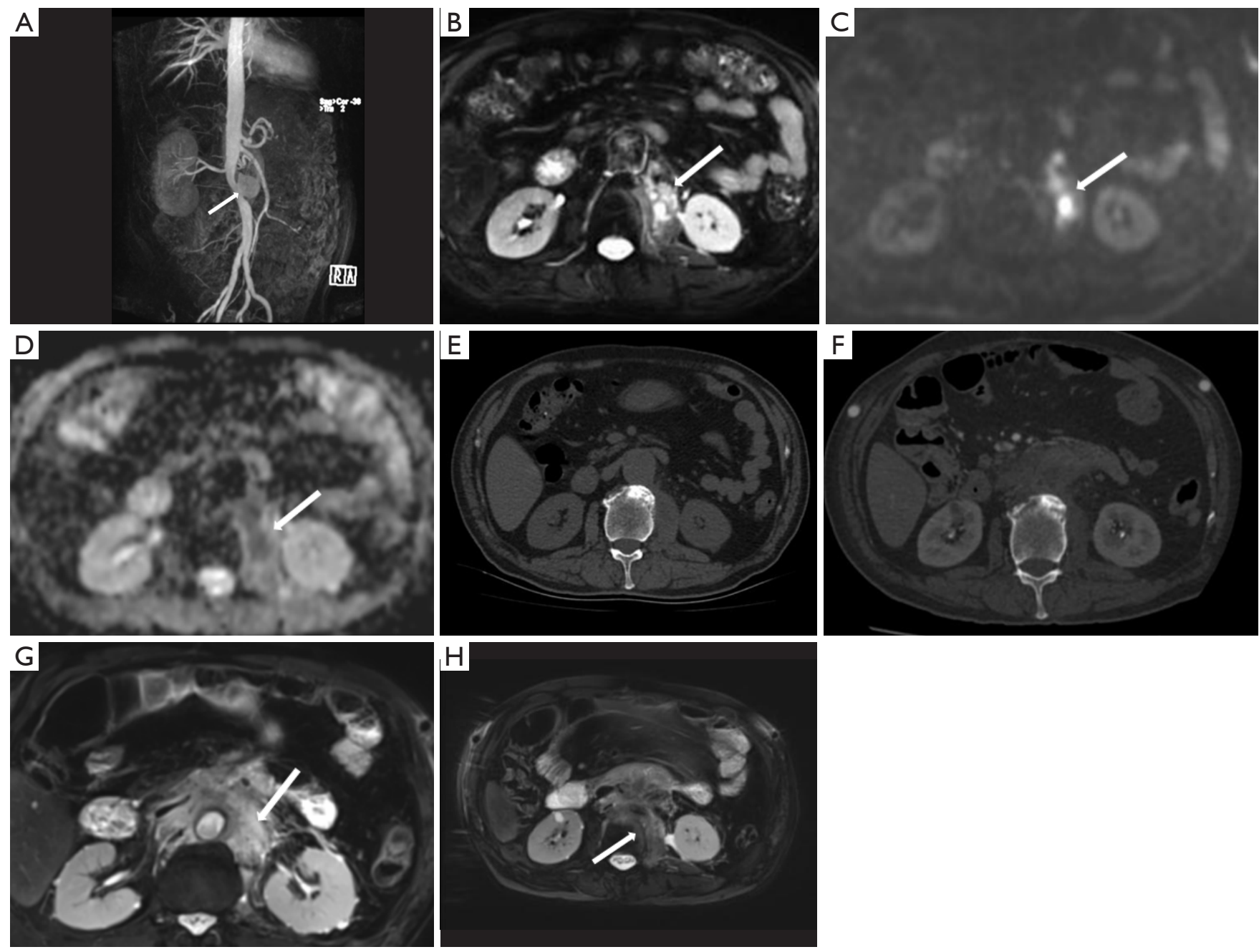

Figure 11 A 69-year-old male with a history of diabetes mellitus presented with fever and abdominal pain. Blood cultures showed Salmonella. (A) 3D MR angiography shows saccular MAA (arrow) at the abdominal aorta. The high signal intensity of the aneurysm wall and anterior left psoas on T2-weighted image (B) was consistent with edema. The central part of the left psoas (arrow) showed significantly high signal intensity on T2-weighted image (B) and DWI (C), low signal intensity on ADC (D), consistent with abscess. High signal intensity of the vertebrae on T2-weighted image indicates edema on the edge of lumbar vertebrae (B) without bone destruction, but with obliteration of fat planes between the vertebral body and left psoas (E). (F-H) During follow-up, the inflammation area was expanded on CT and MR after 4 months (arrow). DWI, diffusion weighted imaging; ADC, apparent diffusion coefficient; MAA, mycotic aortic aneurysm.

of FDG has diagnostic significance for aortoenteric and aortobronchial fistulae.

\section{Follow-up imaging}

Recognition of infection-related complications should be considered during the analysis of follow-up images. Radiological signs are important diagnostic criteria in the detection of aortic graft infection (39), including ectopic gas, peri-graft inflammation, and fluid, thickening of adjacent bowel, pseudoaneurysm formation, and increased ${ }^{18}$ F-FDG uptake at the graft anastomosis (Figure 15) $(13,39)$. The peri-graft fluid within the first 3 months after surgery may contribute to postoperative changes (39). Considering the white blood cell count and clinical symptoms, persistent existence, or gradual increase in the peri-graft fluid should be suspected as an infection-related complication. Extraanatomic bypass procedure avoids 

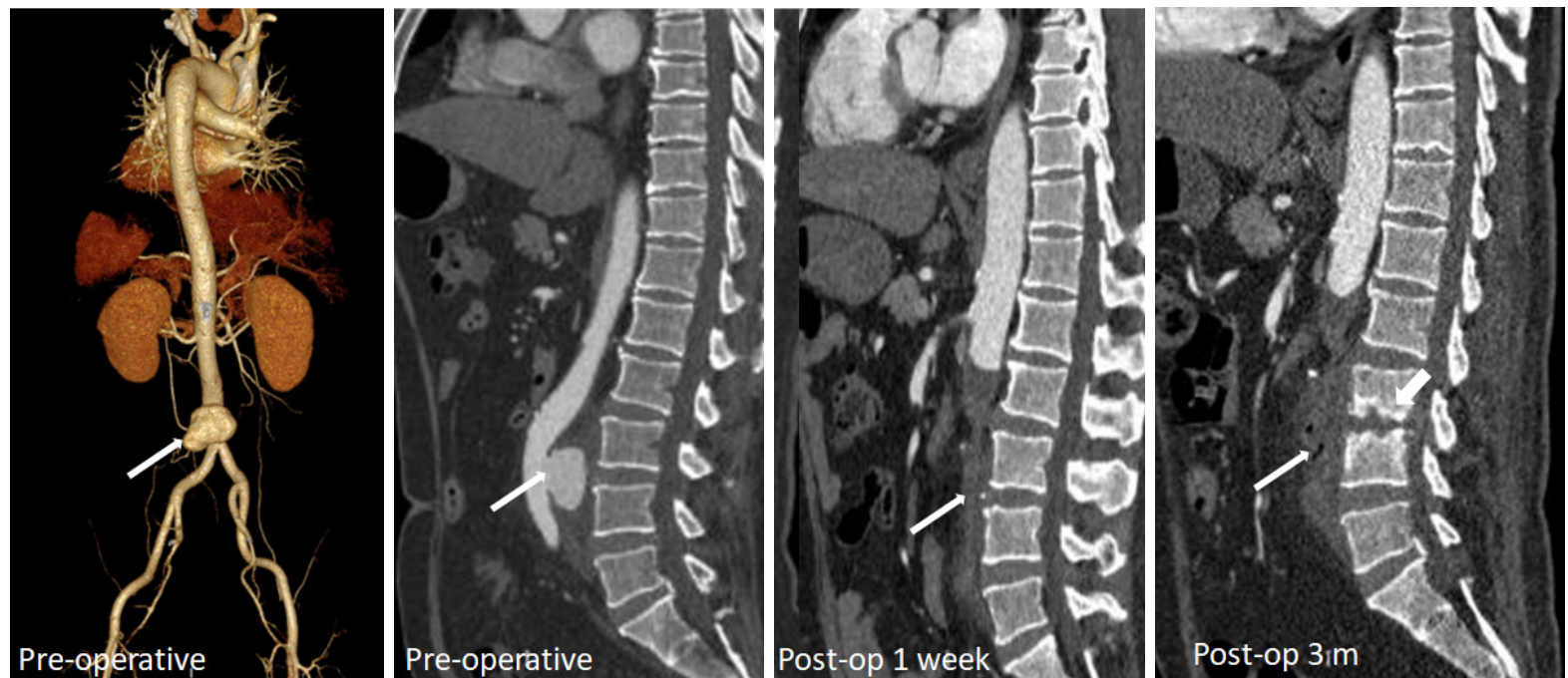

Figure 12 Spondylitis combined with MAAs. A 65-year-old male with a history of diabetes mellitus presented with fever and chest pain. Blood cultures showed KP. Preoperative CTA shows lobular MAA (arrow in 3D view) with normal centrum (arrow in sagittal view). Postoperative CTA shows normal vertebral body with prevertebral soft tissue (arrow in postoperative 1 week sagittal view). CTA after 3 months showed spondylitis short arrow with destruction of adjacent vertebral body endplate, lumbar vertebral space narrow at L3-4 level, and air bubbles in the prevertebral soft tissue (long arrow). CTA, computed tomography angiography; MAAs, mycotic aortic aneurysms.
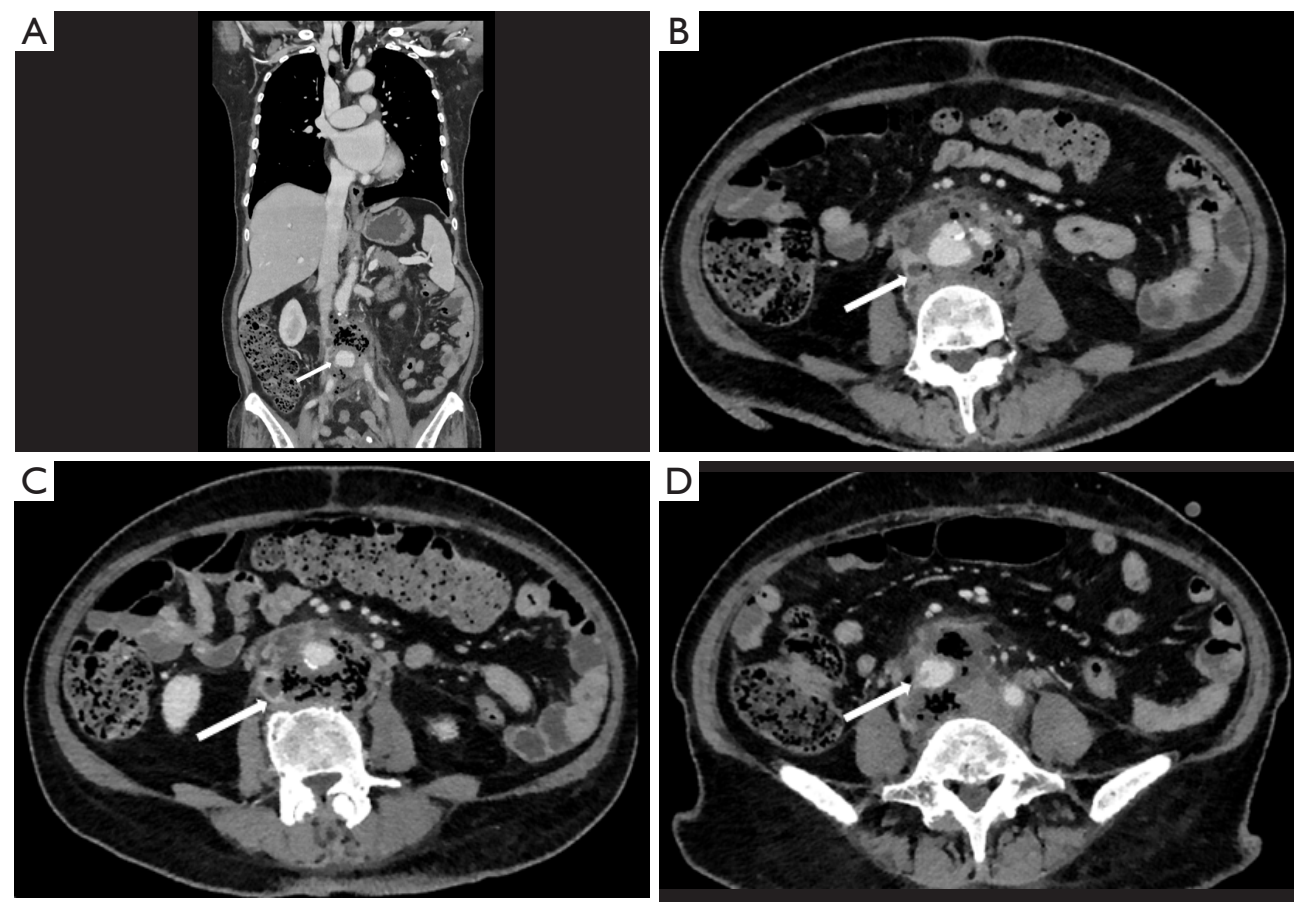

Figure 13 Aortocaval fistula. An 84-year-old female with a history of diabetes mellitus presented with fever, chest pain, and hemoptysis. Blood cultures showed KP. (A) Coronal view of contrast-enhanced CT image shows eccentric pseudoaneurysm (arrow) with adjacent air bubbles and surrounding soft tissue. (B,C) Air bubbles and low-density filling defect (thrombus) (arrow) are shown in the inferior vena cava. Normal anatomical space between the aorta and inferior vena cava has disappeared. (D) Air bubbles and thrombus shown in the right common iliac vein (arrow) with contrast enhancement earlier than that of the left indicates aortocaval fistula. The aortocaval fistula and thrombus in the right common iliac vein and inferior vena cava was confirmed during surgery. 


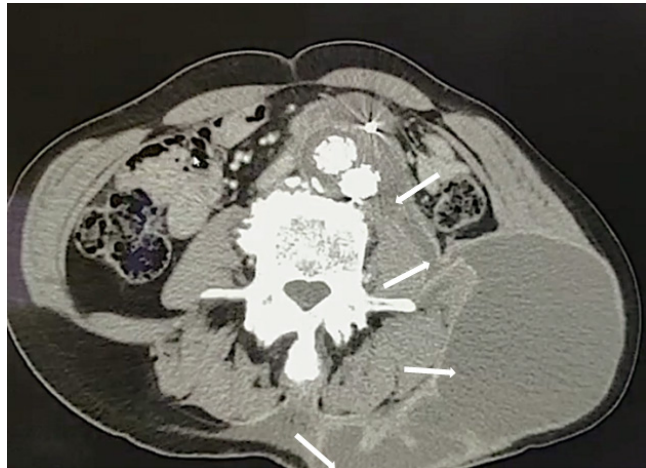

Figure 14 Arteriocutaneous fistulas. A 75 -year-old male with a history of diabetes mellitus and infrarenal pseudoaneurysm, who had undergone endovascular aortic repair 6 months prior, presented with a left hip mass. Contrast-enhanced CT image shows an irregular subcutaneous cyst with rim enhancement connecting with the periaortic fluid. graft placement within an infected field, lowering the risk of graft reinfection compared to in situ reconstruction with a prosthetic graft (17). The endovascular repair of MAAs should be regarded as a temporizing treatment mainly performed on hemodynamically unstable patients. Compared to surgery, the challenges of wide debridement and effective drainage of suppuration brings risks of stent infection (Figure 16), endoleak (Figure 17), aortic fistulas, and potential rupture (8) (Figure 18). Spondylitis and IPA secondary to reinfection occur after both open surgery and endovascular repair (Figures 10,19). A prospective studyincluding 35 patients with suspected aortic graft infectionshowed high concordance between ${ }^{18}$ F-FDG-PET/CT and expert consensus criteria from the Management of Aortic Graft Infection Collaboration (MAGIC) for detecting aortic graft infection (13). Further, ${ }^{18}$ F-FDG-PET/CT can also be used to monitor the response of MAAs and aortic graft
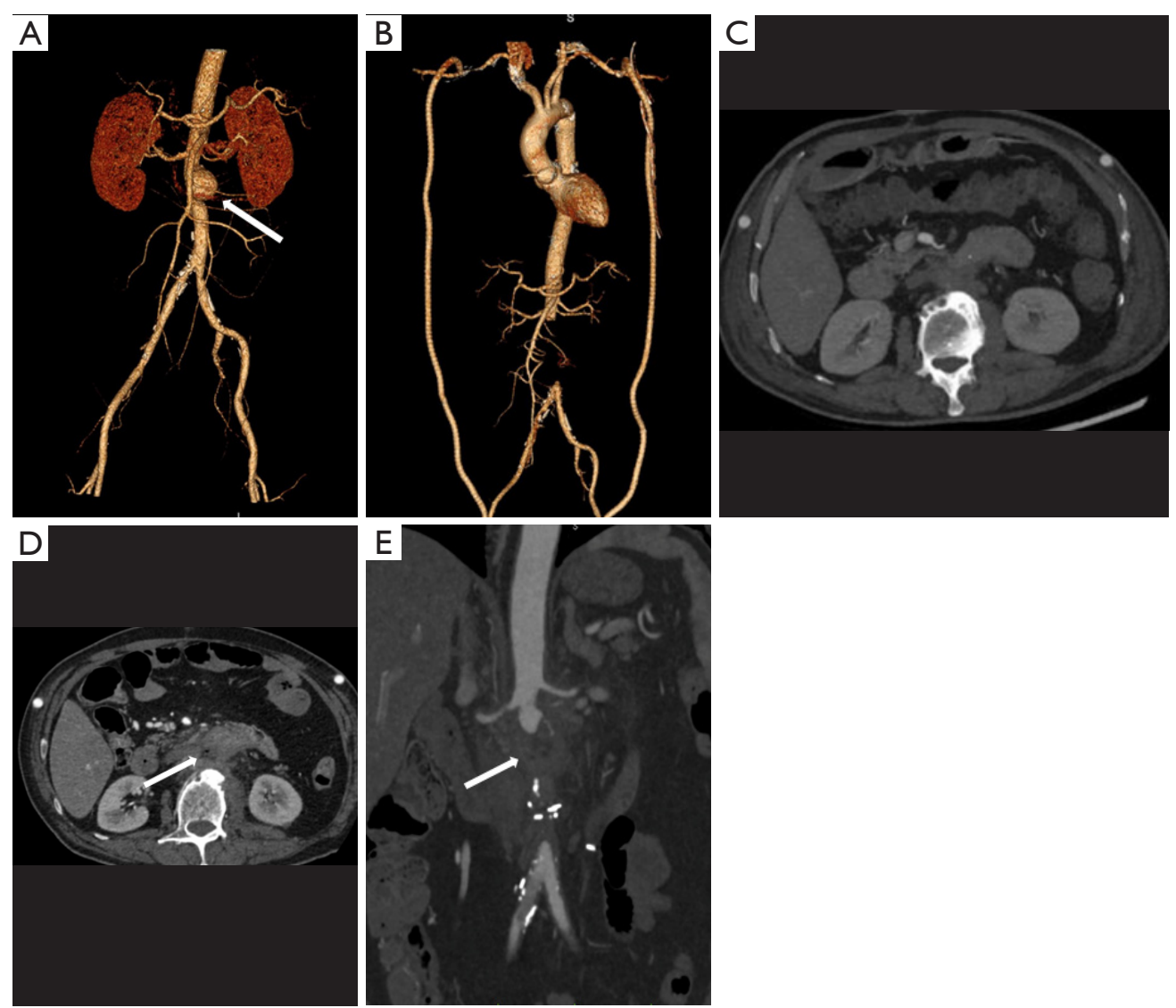

Figure 15 Post-surgical reinfection. A 65-year-old male was diagnosed with mycotic aneurysm caused by Salmonella as shown on 3D CTA (arrow in A). (B,C) The patient underwent open surgery, involving surgical resection of the aneurysm, extensive debridement of the surrounding soft tissue, and extra-anatomic bypass which remained patent after 1 month. (D,E) Transverse and coronal views of follow-up CTA shows soft tissue and air bubbles (arrows) in the native position of the mycotic aneurysm, indicating reinfection. CTA, computed tomography angiography. 

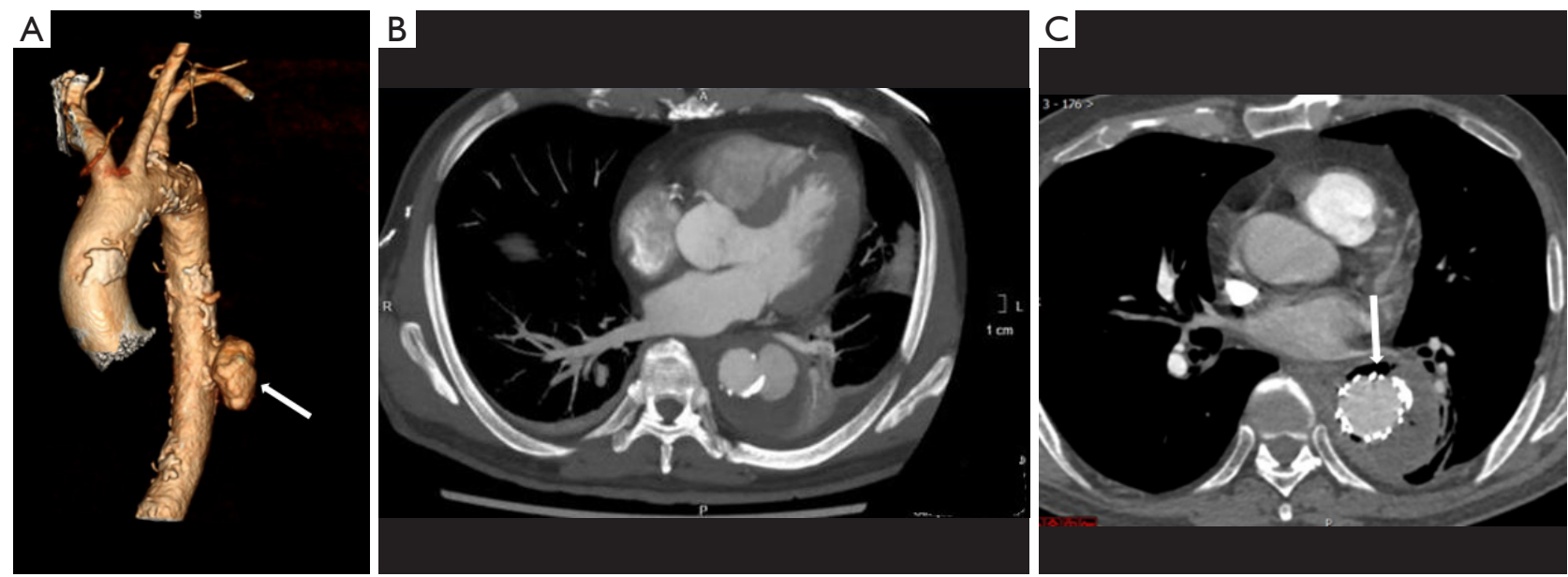

Figure 16 Stent graft infection. (A,B) A 61-year-old male diagnosed with MAAs (arrow) caused by Salmonella, who had undergone EVAR 2 months prior, presented with recurrent fever. (C) Transverse view of follow-up CTA shows an air bubble around the stent graft (arrow). EVAR, endovascular aortic repair; CTA, computed tomography angiography; MAAs, mycotic aortic aneurysms.
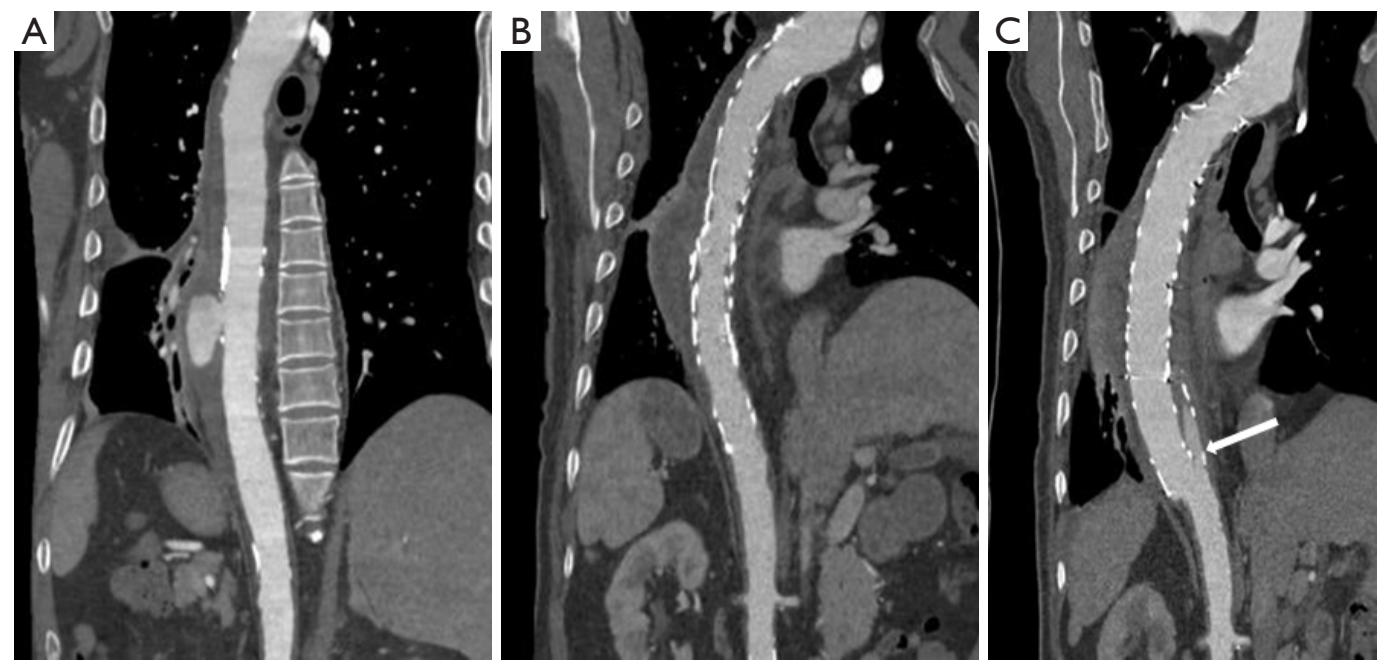

Figure 17 Endoleak after EVAR. A 62-year-old male diagnosed with MAAs caused by Salmonella, who had undergone EVAR, presented with recurrent fever and chest pain. (A) Preoperative CTA image. Curved planar reconstruction (CPR) shows MAA surrounded by soft tissue. (B) CTA image 1 month post-EVAR shows that MAA is successfully occluded but periaortic soft tissue exists. (C) CPR 2 months post-EVAR shows thickened periaortic soft tissue with endoleak at the caudal side of the stent graft (arrow). CTA, computed tomography angiography; EVAR, endovascular aortic aneurysm repair; MAAs, mycotic aortic aneurysms.

infection to antibiotic treatment.

\section{Conclusions}

Diagnosis of life-threatening MAAs is challenging due to the non-specific symptoms and negative blood cultures associated with prior antibiotic use. CT, MRI, and FDG-
PET/CT allow early identification of MAAs, which is crucial for improving patient outcomes. Surveillance imaging permits assessment and promotes treatment efficacy. Figure 20 summarizes both clinical and radiological features that can assist in the diagnosis of MAAs. Clinicians-especially cardiologists, vascular surgeons, and radiologists—-should be familiar with the clinical features 

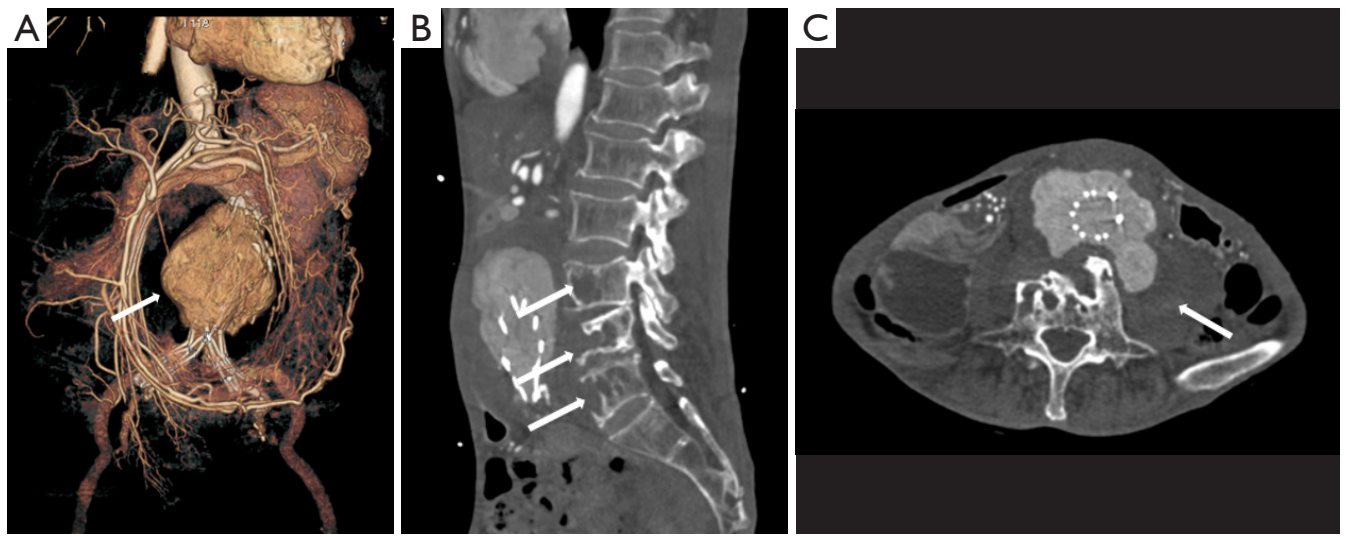

Figure 18 Aneurysm rupture. A 61-year-old male diagnosed with MAAs caused by Mycobacterium tuberculosis, who had undergone EVAR 2 years prior, presented with sudden-onset abdominal pain. (A) 3D CTA shows marked perivascular contrast media extravasation with occupied effect (arrow). (B) Sagittal view of CTA shows destruction of vertebral bodies from L3 to L5 (arrows). (C) Transverse view of CTA shows definite lobular perivascular contrast media extravasation, indicating rupture. A heterogenous, predominantly hypodense tissue existed around the pseudoaneurysm, likely representing subacute hematoma and thrombus (arrow). No fat space is detected between the eroded vertebral body and the pseudoaneurysm. CTA, computed tomography angiography; MAAs, mycotic aortic aneurysms.
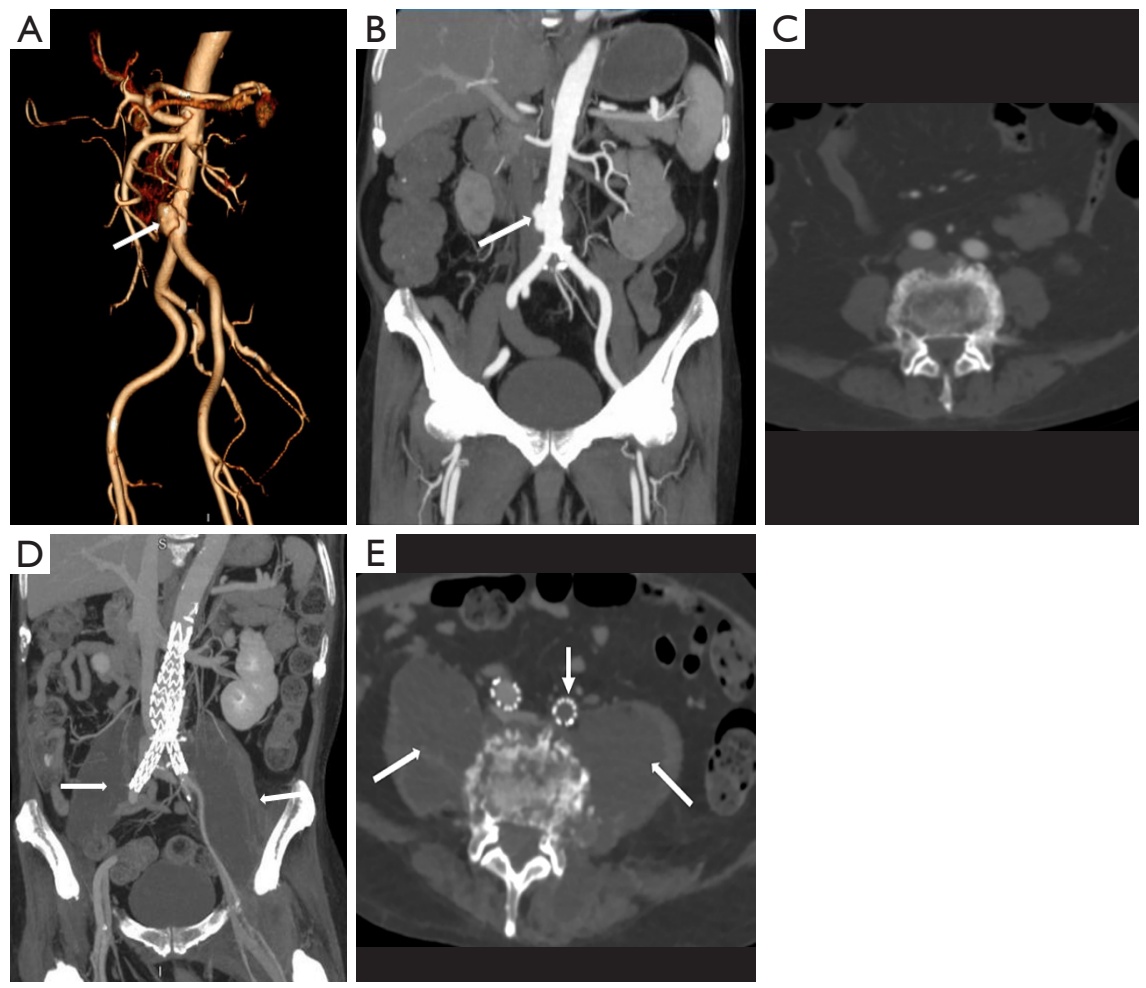

Figure 19 Spondylitis and iliopsoas abscess secondary to reinfection. A 60-year-old male with history of Sweet's syndrome presented with fever and blunt abdominal pain. (A-C) An infrarenal MAA (arrow) is shown on 3D volume rendering image and coronal multiplanar reformatted (MPR) image with normal contour of iliopsoas. Hyperosteogeny was observed at the L5 vertebral body. (D,E) Follow-up CTA at 5 months shows successful exclusion of the MAA. The stent graft in the left common iliac artery is occluded (short arrow in E). Bilateral iliopsoai are swollen with a relatively low-density area with a small bubble (arrows) and contrast-enhanced rim, which support the diagnosis of abscess. The abscess extends to the left erector spinae (arrow in D). Obliteration of fat planes between the vertebrae and iliopsoas, and destruction of the vertebrae indicate spondylitis, is observed. MAA, mycotic aortic aneurysm. 


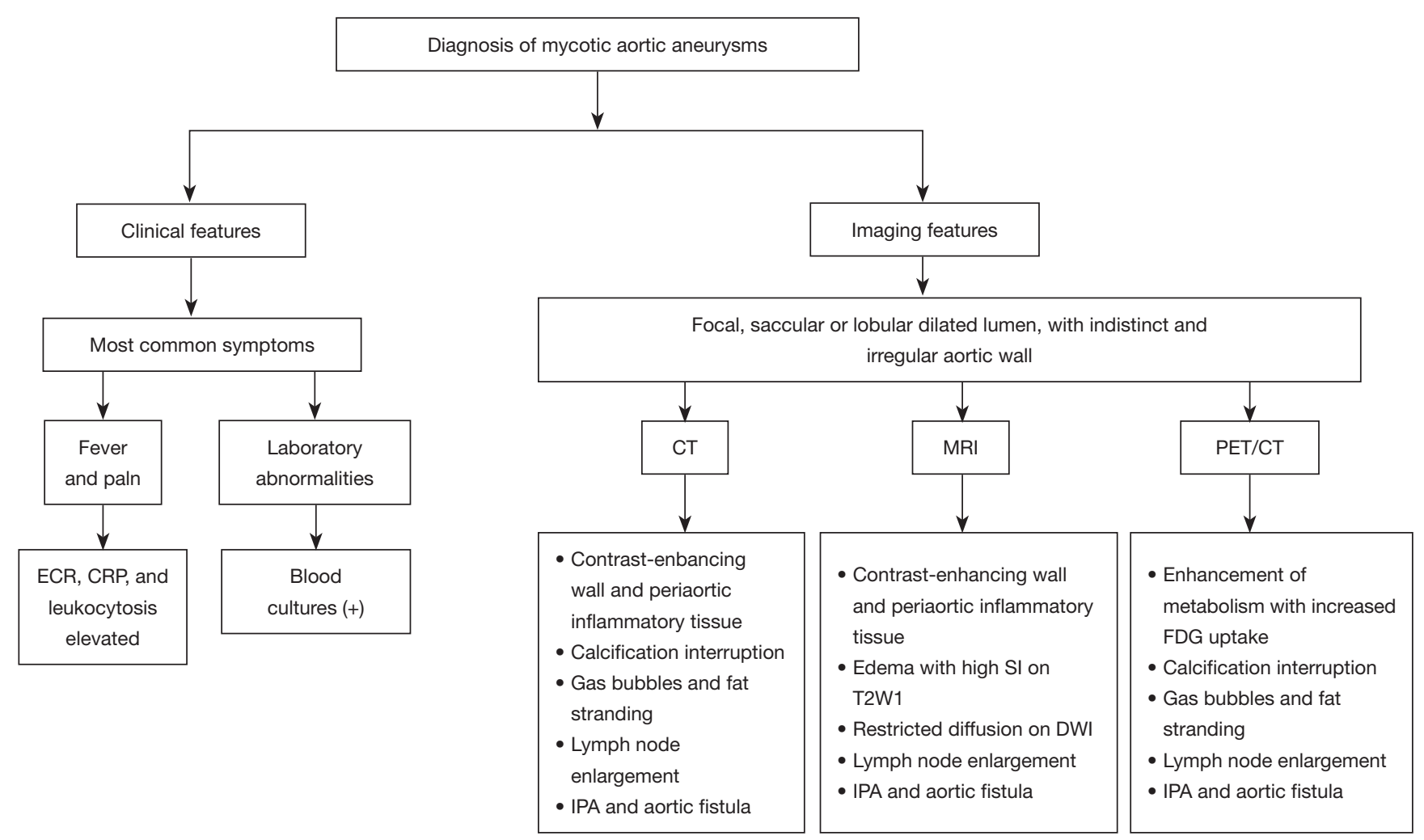

Figure 20 Flow chart showing clinical and radiological features that assist with the diagnosis of mycotic aortic aneurysms. ECR, erythrocyte sedimentation rate; CRP, C-reactive protein; DWI, diffusion weighted imaging; IPA, iliopsoas abscess; SI, signal intensity.

and common manifestations of MAAs to ensure appropriate evaluation and management of the disease.

\section{Acknowledgments}

The authors would like to thank Drs. Fengqiang Wang, Huaiping Yuan and Jin Cheng for their assistance in providing some cases that were used in this study.

Funding: None.

\section{Footnote}

Conflicts of Interest: All authors have completed the ICMJE uniform disclosure form (available at http://dx.doi. org/10.21037/qims-20-941). Dr. ZS serves as an unpaid associate editor of Quantitative Imaging in Medicine and Surgery. The other authors have no conflicts of interest to declare.

Open Access Statement: This is an Open Access article distributed in accordance with the Creative Commons
Attribution-NonCommercial-NoDerivs 4.0 International License (CC BY-NC-ND 4.0), which permits the noncommercial replication and distribution of the article with the strict proviso that no changes or edits are made and the original work is properly cited (including links to both the formal publication through the relevant DOI and the license). See: https://creativecommons.org/licenses/by-nc-nd/4.0/.

\section{References}

1. Osler W. The Gulstonian Lectures, on Malignant Endocarditis. Br Med J 1885;1:522-6.

2. Maleszewski JJ. Inflammatory ascending aortic disease: perspectives from pathology. J Thorac Cardiovasc Surg 2015;149:S176-83.

3. Stone JR, Bruneval P, Angelini A, Bartoloni G, Basso C, Batoroeva L, Buja LM, Butany J, d'Amati G, Falloon JT, Gittenberger-de Groot AC, Gouveia RH, Halushka MK, Kelly KL, Kholova I, Leone O, Litovsku SH, Maleszewski JJ, Miller DV, Mitchell RN, Preston SD, Pucci A, Radio SJ, Rodriguez ER, Sheppard MN, Suvarna SK, Tan 
CD, Thiene G, van der Wal AC, Veinot JP. Consensus statement on surgical pathology of the aorta from the Society for Cardiovascular Pathology and the Association for European Cardiovascular Pathology: I. Inflammatory diseases. Cardiovasc Pathol 2015;24:267-78.

4. Buckmaster MJ, Curci JA, Murray PR, Liao S, Allen BT, Sicard GA, Thompson RW. Source of elastin-degrading enzymes in mycotic aortic aneurysms: bacteria or host inflammatory response? Cardiovasc Surg 1999;7:16-26.

5. McCready RA, Bryant MA, Divelbiss JL, Chess BA, Chitwood RW, Paget DS. Arterial infections in the new millenium: an old problem revisited. Ann Vasc Surg 2006;20:590-5.

6. Carreras M, Larena JA, Tabernero G, Langara E, Pena JM. Evolution of Salmonella aortitis towards the formation of abdominal aneurysm. Eur Radiol 1997;7:54-6.

7. Maeda H, Umezawa H, Goshima M, Hattori T, Nakamura T, Umeda T, Shiono M. Primary infected abdominal aortic aneurysm: surgical procedures, early mortality rates, and a survey of the prevalence of infectious organisms over a 30year period. Surg Today 2011;41:346-51.

8. Plotkin A, Magee GA, Elsayed RS, Byerly S, Ham SW, Han SM, Manzur MF, Rowe VL, Weaver FA. Methicillinresistant Staphylococcus aureus portends a poor prognosis after endovascular repair of mycotic aortic aneurysms and aortic graft infections. J Vasc Surg 2020;72:276-85.

9. Sörelius K, di Summa PG. On the Diagnosis of mycotic aortic aneurysms. Clin Med Insights Cardiol 2018;12:1179546818759678.

10. Jones KG, Bell RE, Sabharwal T, Aukett M, Reidy JF, Taylor PR. Treatment of mycotic aortic aneurysms with endoluminal grafts. Eur J Vasc Endovasc Surg 2005;29:139-44.

11. Patel HJ, Williams DM, Upchurch GR Jr, Dasika NL, Eliason JL, Deeb GM. Thoracic aortic endovascular repair for mycotic aneurysms and fistulas. J Vasc Surg 2010;52:37S-40S.

12. Husmann L, Huellner MW, Ledergerber B, Eberhard N, Kaelin MB, Anagnostopoulos A, Kudura K, Burger IA, Mestres CA, Rancic Z, Hasse B; Vasgra Cohort. Diagnostic accuracy of PET/CT and contrast enhanced CT in patients with suspected infected aortic aneurysms. Eur J Vasc Endovasc Surg 2020;59:972-81.

13. Dong W, Li Y, Zhu J, Xia J, He L, Yun M, Jiao J, Zhu G, Hacker M, Wei Y, Zhang X, Li X. Detection of aortic prosthetic graft infection with $18 \mathrm{~F}-\mathrm{FDG}$ PET/ CT imaging, concordance with consensus MAGIC graft infection criteria. J Nucl Cardiol 2020. doi: 10.1007/ s12350-020-02227-9.

14. Silvestri V, Ettorre GD, Borrazzo C, Mele R. Many Different Patterns under a Common Flag: Aortic Pathology in HIV-A Review of Case Reports in Literature. Ann Vasc Surg 2019;59:268-84.

15. Pillsbury MM, Geha RM, Edson RS. Sticky Business: a syndrome of mucoid bacterial spread. BMJ Case Rep 2019;12:e226956.

16. Luo Y, Zhu J, Dai X, Fan H, Feng Z, Zhang Y, Hu F. Endovascular treatment of primary mycotic aortic aneurysms: a 7-year single-center experience. J Int Med Res 2018;46:3903-9.

17. Kyriakides C, Kan Y, Kerle M, Cheshire NJ, Mansfield AO, Wolfe JH. 11-year experience with anatomical and extra-anatomical repair of mycotic aortic aneurysms. Eur J Vasc Endovasc Surg 2004;27:585-9.

18. Sorelius K, Mani K, Bjorck M, Sedivy P, Wahlgren CM, Taylor P, Clough RE, Lyons O, Thompson M, Brownrigg J, Ivancev K, Davis M, Jenkins MP, Jaffer U, Bown M, Rancic Z, Mayer D, Brunkwall J, Gawenda M, Kolbel T, Jean-Baptiste E, Moll F, Berger P, Liapos CD, Moulakakis KG, Langenskiold M, Roos H, Larzon T, Pirouzram A. Wanhainen A for the European MAA collaborators. Endovascular treatment of mycotic aortic aneurysms: a European multicenter study. Circulation 2014;130:2136-42.

19. Huang YK, Chen CL, Lu MS, Tsai FC, Lin PL, Wu CH, Chiu CH. Clinical, microbiologic, and outcome analysis of mycotic aortic aneurysm: the role of endovascular repair. Surg Infect (Larchmt) 2014;15:290-8.

20. Kan CD, Lee HL, Yang YJ. Outcome after endovascular stent graft treatment for mycotic aortic aneurysm: a systematic review. J Vasc Surg 2007;46:906-12.

21. Chiu CH, Su LH, Chu C. Salmonella enterica serotype Choleraesuis: Epidemiology, pathogenesis, clinical disease, and treatment. Clin Microbiol Rev 2004;17:311-22.

22. Guiney DG. The role of host cell death in Salmonella infections. Curr Top Microbiol Immunol 2005;289:131-50.

23. Moore KJ, Sheedy FJ, Fisher EA. Macrophages in atherosclerosis: a dynamic balance. Nat Rev Immunol 2013;13:709-21.

24. Siu LK, Yeh KM, Lin JC, Fung CP, Chang FY. Klebsiella pneumoniae liver abscess: a new invasive syndrome. Lancet Infect Dis 2012;12:881-7.

25. Shon AS, Bajwa RP, Russo TA. Hypervirulent (hypermucoviscous) Klebsiella pneumoniae: a new and dangerous breed. Virulence 2013;4:107-18.

26. Long R, Guzman R, Greenberg H, Safneck J, Hershfield E. Tuberculous mycotic aneurysm of the aorta: review 
of published medical and surgical experience. Chest 1999;115:522-31.

27. Shigemitsu O, Hadama T, Miyamoto S, Anai H, Sako $\mathrm{H}$. Tuberculous pseudoaneurysm of the ascending aorta with intracranial tuberculoma. J Cardiovasc Surg (Torino) 2002;43:59-62.

28. Ting AC, Cheng SW. Femoral pseudoaneurysms in drug addicts. World J Surg 1997;21:783-6.

29. Lee WK, Mossop PJ, Little AF, Fitt GJ, Vrazas JI, Hoang JK, Hennessy OF. Infected (mycotic) aneurysms: Spectrum of imaging appearances and management. Radiographics 2008;28:1853-68.

30. Ramachandran Nair H, Goura P, Pitchai S, Madathipat U. Brucella-induced ruptured infrarenal dissecting abdominal aortic aneurysm. Aorta (Stamford) 2019;7:56-8.

31. Huang JJ, Ruaan MK, Lan RR, Wang MC. Acute pyogenic iliopsoas abscess in Taiwan: clinical features, diagnosis, treatments and outcome. J Inf Secur 2000;40:248-55.

32. Hsieh MS, Huang SC, Loh EW, Tsai CA, Hung YY, Tsan YT, Huang JA, Wang LM, Hu WY. Features and treatment modality of iliopsoas abscess and its outcome: a 6-year hospital-based study. BMC Infect Dis 2013;13:578.

33. Lai YC, Lin PC, Wang WS, Lai JI. An update on psoas muscle abscess: an 8- year experience and review of literature. Int J Gerontol 2011;5:75-9.

34. Hu SY, Hsieh MS, Chang YT, Huang CC, Tsai CA,

Cite this article as: Zhang N, Xiong W, Li Y, Mao Q, $\mathrm{Xu} \mathrm{S}$, Zhu J, Sun Z, Sun L. Imaging features of mycotic aortic aneurysms. Quant Imaging Med Surg 2021;11(6):2861-2878. doi: 10.21037/qims-20-941
Tsai CL, Hsu CY, Shen CH, Chang YZ. Clinical features, management, and outcome of iliopsoas abscess associated with cardiovascular disorders: a hospital- based observational case series study. BMC Musculoskelet Disord 2019;20:474.

35. Nakamura T, Morimoto T, Katsube K, Yamamori Y, Mashino J, Kikuchi K. Clinical characteristics of pyogenic spondylitis and psoas abscess at a tertiary care hospital: a retrospective cohort study. J Orthop Surg Res 2018;13:302.

36. Saers SJ, Scheltinga MR. Primary aortoenteric fistulae. $\mathrm{Br}$ J Surg 2005;92:143-52.

37. Bergqvist D, Björck M. Secondary arterioenterc fistulation-a systematic literature analysis. Eur J Endovasc Surg 2009;37:31-42.

38. Karhof S, van Roeden SE, Oosterheert JJ, Bleeker-Rovers CP, Renders NHM, de Borst GJ, Kampschreur LM, Hoepelman AIM, Koning OHJ, Wever PC. Primary and secondary arterial fistulas during chronic Q fever. J Vasc Surg 2018;68:1906-13.

39. Lyons OT, Baguneid M, Barwick TD, Bell RE, Foster N, Homer-Vanniasinkam S, Hopkins S, Hussain A, Katsanos K, Modarai B, Sandoe JA, Thomas S, Price NM. Diagnosis of Aortic Graft Infection: A Case Definition by the Management of Aortic Graft Infection Collaboration (MAGIC). Eur J Vasc Endovasc Surg 2016;52:758-63. 